\title{
О ТЕРМОДИНАМИЧЕСКОИ ВЕРОЯТНОСТИ ПОЛУЧЕНИЯ СУЛЬФИДА КАЛЬЦИЯ ИЗ ФОСФОГИПСА
}

\author{
(Представил М. Вейдерма)
}

Термодинамические закономерности термического разложения сульфата кальция в присутствии твердых или газовых восстановителей изучались многими исследователями, занимающимися проблемой утилизации фосфогипса - многотоннажных отходов производства экстракционной фосфорной кислоты. В оєновном ограничивались расчетом свободной энергии Гиббса $\left(\Delta G_{T}^{0}\right)$, не учитывая реальный состав газовой среды, образующейся при неполном сжигании углеводородного топлива $\left[{ }^{1-6}\right]$. Изучение влияния состава газовой среды на процесс восстановления сульфата кальция до диоксида серы проведено в [ $\left.{ }^{7}\right]$ с целью нахождения условий для предотвращения образования сульфида кальция. Более подробно процесс рассмотрен в $\left.{ }^{8}\right]$ с построением диаграммы состояния и определением на ней областей существования разных твердых продуктов в зависимости от состава исходной газовой смеси и парциального давления выделяющегося диоксида серы. Однако реакции, выбранные для определения термодинамических характеристик, описывают процесс лишь в области высоких температур с получением оксида кальция и диоксида серы. Для описания низкотемпературной области процесса восстановления сульфата кальция с получением $\mathrm{CaS}$ необходим дополнительный термодинамический анализ большего числа реакций. Кроме того, в опубликованных работах практически не затронут вопрос получения $\mathrm{CaS}$ с минимизацией образования $\mathrm{CaO}$. Этот вариант процесса представляет практический интерес, поскольку сульфид кальция может быть применен в промышленности цветных металлов в качестве сульфидизатора, или сырья для получения элементарной серы.

Для определения термодинамической вероятности получения сульфида кальция в процессе восстановительного разложения сульфата кальция при неполном сжигании природного газа проведен термодинамический анализ систем $\mathrm{CaSO}_{4}-\mathrm{H}_{2}-\mathrm{H}_{2} \mathrm{O}, \mathrm{CaSO}_{4}-\mathrm{CO}-\mathrm{CO}_{2}$ и $\mathrm{CaSO}_{4}-$ $-\mathrm{C}-\mathrm{O}_{2}$ в температурном интервале $800-1400 \mathrm{~K}$ на базе 22 конкурирующих реакций.

Восстановление водородом:

$$
\begin{aligned}
& \mathrm{CaSO}_{4}(\mathrm{~T})+4 \mathrm{H}_{2}(\mathrm{r}) \rightleftarrows \mathrm{CaS}(\mathrm{T})+4 \mathrm{H}_{2} \mathrm{O}(\mathrm{r}), \\
& \mathrm{CaSO}_{4}(\mathrm{~T})+3 \mathrm{H}_{2}(\mathrm{r}) \rightleftharpoons \mathrm{CaO}(\mathrm{r})+3 \mathrm{H}_{2} \mathrm{O}(\mathrm{r})+\frac{1}{2} \mathrm{~S}_{2}(\mathrm{r}), \\
& \mathrm{CaSO}_{4}(\mathrm{~T})+\mathrm{H}_{2}(\mathrm{r}) \rightleftharpoons \mathrm{CaO}(\mathrm{T})+\mathrm{H}_{2} \mathrm{O}(\mathrm{r})+\mathrm{SO}_{2}(\mathrm{r}), \\
& \mathrm{CaO}(\mathrm{T})+3 \mathrm{H}_{2}(\mathrm{r})+\mathrm{SO}_{2}(\mathrm{r}) \rightleftharpoons \mathrm{CaS}(\mathrm{T})+3 \mathrm{H}_{2} \mathrm{O}(\mathrm{r}), \\
& \mathrm{SO}_{2}(\mathrm{r})+2 \mathrm{H}_{2}(\mathrm{r}) \rightleftharpoons 2 \mathrm{H}_{2} \mathrm{O}(\mathrm{r})+\frac{1}{2} \mathrm{~S}_{2}(\mathrm{r}) .
\end{aligned}
$$

Восстановление оксидом углерода:

$$
\begin{gathered}
\mathrm{CaSO}_{4}(\mathrm{~T})+4 \mathrm{CO}(\mathrm{r}) \rightleftharpoons \mathrm{CaS}(\mathrm{\tau})+4 \mathrm{CO}_{2}(\mathrm{r}) \\
\mathrm{CaSO}_{4}(\mathrm{~T})+3 \mathrm{CO}(\mathrm{r}) \rightleftharpoons \mathrm{CaO}(\mathrm{T})+3 \mathrm{CO}_{2}(\mathrm{r})+\frac{1}{2} \mathrm{~S}_{2}(\mathrm{r}),
\end{gathered}
$$




$$
\begin{aligned}
& \mathrm{CaSO}_{4}(\mathrm{~T})+\mathrm{CO}(\mathrm{r}) \rightleftarrows \mathrm{CaO}(\mathrm{T})+\mathrm{CO}_{2}(\mathrm{r})+\mathrm{SO}_{2}(\mathrm{r}), \\
& \mathrm{CaO}(\mathrm{T})+3 \mathrm{CO}(\mathrm{r})+\mathrm{SO}_{2}(\mathrm{r}) \rightleftharpoons \mathrm{CaS}(\mathrm{r})+3 \mathrm{CO}_{2}(r), \\
& \mathrm{SO}_{2}(\mathrm{r})+2 \mathrm{CO}(\mathrm{r}) \rightleftarrows 2 \mathrm{CO}_{2}(\mathrm{r})+\frac{1}{2} \mathrm{~S}_{2}(\mathrm{r}) .
\end{aligned}
$$

Восстановление углеродом:

$$
\begin{aligned}
& \mathrm{CaSO}_{4}(\mathrm{~T})+4 \mathrm{C}(\mathrm{T}) \rightleftarrows \mathrm{CaS}(\mathrm{T})+4 \mathrm{CO}(\mathrm{r}), \\
& \mathrm{CaSO}_{4}(\mathrm{~T})+2 \mathrm{C}(\mathrm{T}) \rightleftarrows \mathrm{CaS}(\mathrm{T})+2 \mathrm{CO}_{2}(\mathrm{r}), \\
& 2 \mathrm{CaSO}_{4}(\mathrm{~T})+\mathrm{C}(\mathrm{T}) \rightleftarrows 2 \mathrm{CaO}(\mathrm{T})+\mathrm{CO}_{2}(\mathrm{r})+2 \mathrm{SO}_{2}(\mathrm{r}), \\
& \mathrm{CO}_{2}(\mathrm{r})+\mathrm{C}(\mathrm{T}) \rightleftarrows 2 \mathrm{CO}(\mathrm{r}), \\
& \mathrm{SO}_{2}(\mathrm{r})+\mathrm{C}(\mathrm{T}) \rightleftarrows \mathrm{CO}_{2}(\mathrm{r})+\frac{1}{2} \mathrm{~S}_{2}(\mathrm{r}), \\
& \mathrm{SO}_{2}(\mathrm{r})+2 \mathrm{C}(\mathrm{T}) \rightleftarrows 2 \mathrm{CO}(\mathrm{r})+\frac{1}{2} \mathrm{~S}_{2}(\mathrm{r}) .
\end{aligned}
$$

Реакции окисления:

$$
\begin{aligned}
& \mathrm{H}_{2}(\mathrm{r})+\frac{1}{2} \mathrm{O}_{2}(\mathrm{r}) \rightleftarrows \mathrm{H}_{2} \mathrm{O}(\mathrm{r}), \\
& \mathrm{CO}(\mathrm{r})+\frac{1}{2} \mathrm{O}_{2}(\mathrm{r}) \rightleftarrows \mathrm{CO}_{2}(\mathrm{r}), \\
& \mathrm{C}(\mathrm{T})+\frac{1}{2} \mathrm{O}_{2}(\mathrm{r}) \rightleftarrows \mathrm{CO}(\mathrm{r}), \\
& \mathrm{CaS}(\mathrm{T})+2 \mathrm{O}_{2}(\mathrm{r}) \rightleftarrows \mathrm{CaSO}_{4}(\mathrm{~T}), \\
& \mathrm{CaS}(\mathrm{T})+\frac{3}{2} \mathrm{O}_{2}(\mathrm{r}) \rightleftarrows \mathrm{CaO}^{(\mathrm{T})}+\mathrm{SO}_{2}(\mathrm{r}), \\
& \frac{1}{4} \mathrm{CaS}(\mathrm{T})+\frac{3}{4} \mathrm{CaSO}_{4}(\mathrm{~T}) \rightleftarrows \mathrm{CaO}^{(\mathrm{T})}+\mathrm{SO}_{2}(\mathrm{r}) .
\end{aligned}
$$

Вероятность протекания реакций оценивали по равновесным парциальным давлениям продуктов реакций, а также по значению $\Delta G_{T}$ с учетом реальных парциальных давлений газов согласно уравнению

$$
\Delta G_{T}=\Delta G_{T}^{0}+R T \ln \prod_{i}^{n} p_{i}^{0 \pm v t},
$$

где $\Delta G_{T}^{0}-$ свободная энергия Гиббса, $p_{i}^{0}-$ исходное парциальное давление газового компонента, $\pm v_{i}-$ стехиометрический коэффициент в уравнении реакции.

Расчет $\Delta G_{T}^{0} \quad$ был проведен по методу Темкина-Шварцмана. Равновесные парциальные давления газовых компонентов реакций рассчитывали на компьютере по алгоритму, описанному в [ $\left.{ }^{9}\right]$. Принято, что газовая фаза описывается уравнением идеального газа и активности твердых компонентов реакций равны единице. Наибольший интерес представляла система со следующими парциальными давлениями исходных газовых компонентов, атм.:

$$
\begin{aligned}
& p_{\mathrm{H}_{2}}^{0}: p_{\mathrm{H}: \mathrm{O}}^{0}=0,01: 0,3 ; 0,2: 0,01 ; 0,2: 0,2 . \\
& p_{\mathrm{CO}}^{0}: p_{\mathrm{CO}_{2}}^{0}=0,01: 0,3 ; 0,2: 0,01 ; 0,2: 0,1 . \\
& p_{\mathrm{SO}_{2}}^{0}=0,005 . \\
& p_{\mathrm{S}_{2}}^{0}=0,001 . \\
& p_{\mathrm{O}_{2}}^{0}=10^{-10}
\end{aligned}
$$



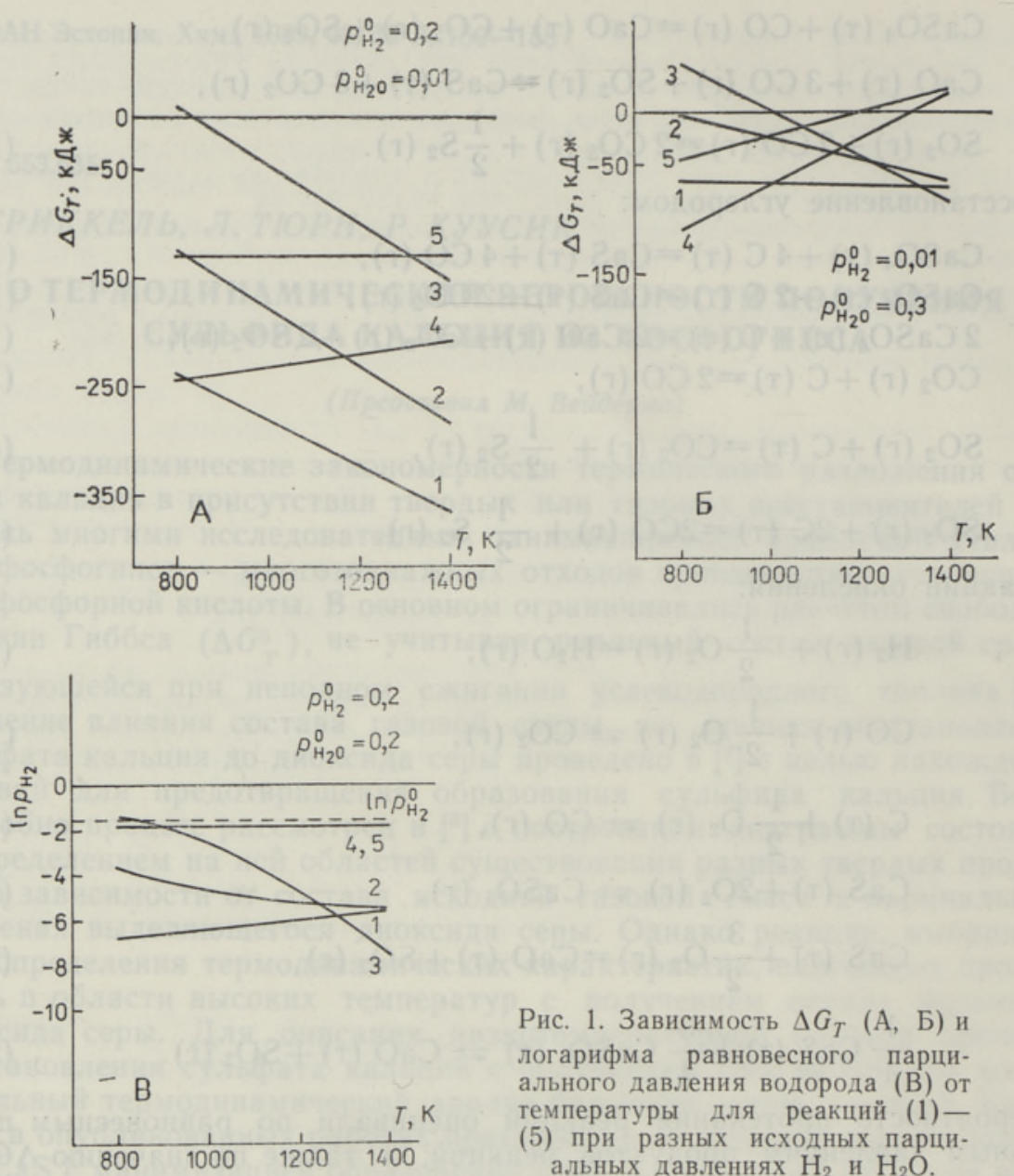

Рис. 1. Зависимость $\Delta G_{T}(\mathrm{~A}$, Б) и логарифма равновесного парциального давления водорода (В) от температуры для реакций (1)(5) при разных исходных парциальных давлениях $\mathrm{H}_{2}$ и $\mathrm{H}_{2} \mathrm{O}$.

Последний состав соответствует среднему составу газовой фазы в зоне реакции при восстановлении сульфата кальция газами, полученными при неполном сжигании метана с коэффициентом расхода воздуха $0,55-0,65$, и принят в основу расчетов $\Delta G_{T}$ и равновесных парциальных давлений.

Изменение $\Delta G_{T}$ в зависимости от температуры и состава газовой фазы для реакций с участием водорода показано на рис. 1, А и Б. В соответствии со значениями $\Delta G_{T}$ при высоком восстановительном потенциале $p_{\mathrm{H}_{2}}^{0}=0,2$ и $p_{\mathrm{H}_{2} \mathrm{O}}^{0}=0,01$ во всем исследованном температурном интервале преобладает реакция образования сульфида кальция (1). Снижение восстановительного потенциала приводит к уменьшению температурной зависимости $\Delta G_{\tau}$ для реакции (1). Например, при $p_{\mathrm{H}_{2}}^{0}=0,01$ и $p_{\mathrm{H}_{2} \mathrm{O}}^{0}=0,3$ выше $1350 \mathrm{~K}$ термодинамически более вероятным становится образование диоксида серы по реакции (3). Возможным становится также окисление сульфида кальция водяным паром по обратной реакции (4), характеризующейся сравнительно высокой температурной зависимостью $\Delta G_{T}$. При восстановлении сульфата кальция в реальной газовой среде $\left(p_{\mathrm{H}_{2}}^{0}=0,2\right.$ и $\left.\quad p_{\mathrm{H}_{2} \mathrm{O}}^{0}=0,2\right)$ наименьшее равновесное парциальное давление его соответствует реакции образования сульфида кальция (1) (рис. 1, В). Выше $1300 \mathrm{~K}$ наиболее вероятной становится реакция образования оксида кальция по (3), а парциальное давление водорода, образующегося по реакции (1), имеет ту же величину, что и для реакции 

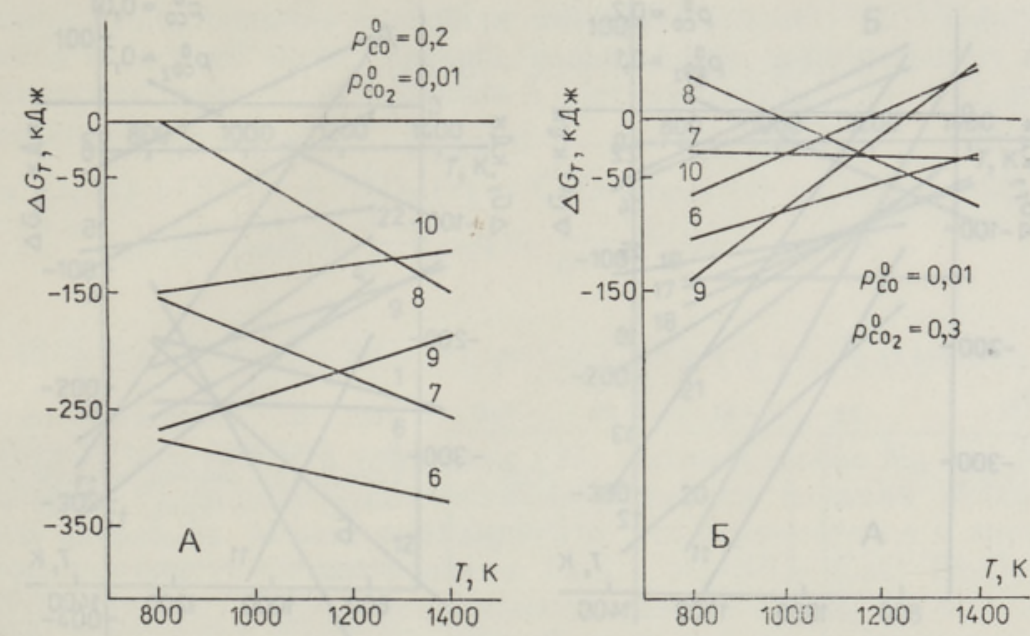

Рис. 2. Зависимость $\Delta G_{T}(\mathrm{~A}$, Б) и логарифма равновесного парциального давления оксида углерода (B) от температуры для реакций (6)-(10) при разных исходных парциальных давлениях $\mathrm{CO}$ и $\mathrm{CO}_{2}$.

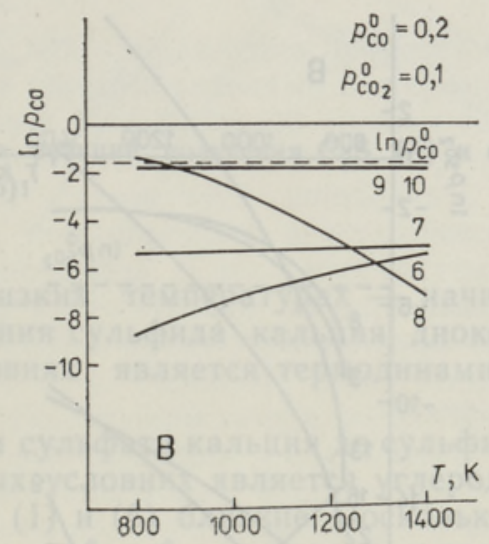

получения серы (2). При этом изменение равновесного давления водорода для реакции (3) характеризуется наибольшей температурной зависимостью и выделение диоксида серы является термодинамически возможным уже при $880 \mathrm{~K}$. Это означает, что в данных условиях возможно одновременное образование $\mathrm{SO}_{2}$ и $\mathrm{CaS}$.

Уменьшение содержания в восстановительных газах диоксида углерода, как следует из сравнения рис. 2 , А и Б, способствует протеканию процесса в направлении образования сульфида кальция. Наоборот, повышение концентрации диоксида углерода в ходе процесса и уменьшение концентрации оксида углерода приводят к уменьшению вероятности протекания реакции (6) и при $p_{\mathrm{Co}}^{0}=0,01$ и $p_{\mathrm{CO}_{2}}^{0}=0,3$ выше $1280 \mathrm{~K}$ пресбладающим становится выделение диоксида серы по релкции (8) (рис. 2, Б). Наименьшее равновесное давление оксида угл рс ла з случае сго реального содержания в исходной газовой смеси ( $p_{\mathrm{Co}}^{0}=0,2$ и $\left.p_{\mathrm{CO}_{2}}^{0}=0,1\right)$ имеет место при $800 \mathrm{~K}$ и соответствует реакции образования сульфида кальция (6) (рис. 2, В). Начало образования $\mathrm{CaO}$ и $\mathrm{SO}_{2}$ возможно при $900 \mathrm{~K}$, реакция (8) становится прєобладающей выше $1300 \mathrm{~K}$.

Влияние температуры и состава исходной газовой смеси на вероятность протекания реакции с участием твердого восстановителя показано на рис. 3. Во всем исследованном интервале температур преобладают реакции (11) и (12) с образованием сульфида кальция. Причем более 

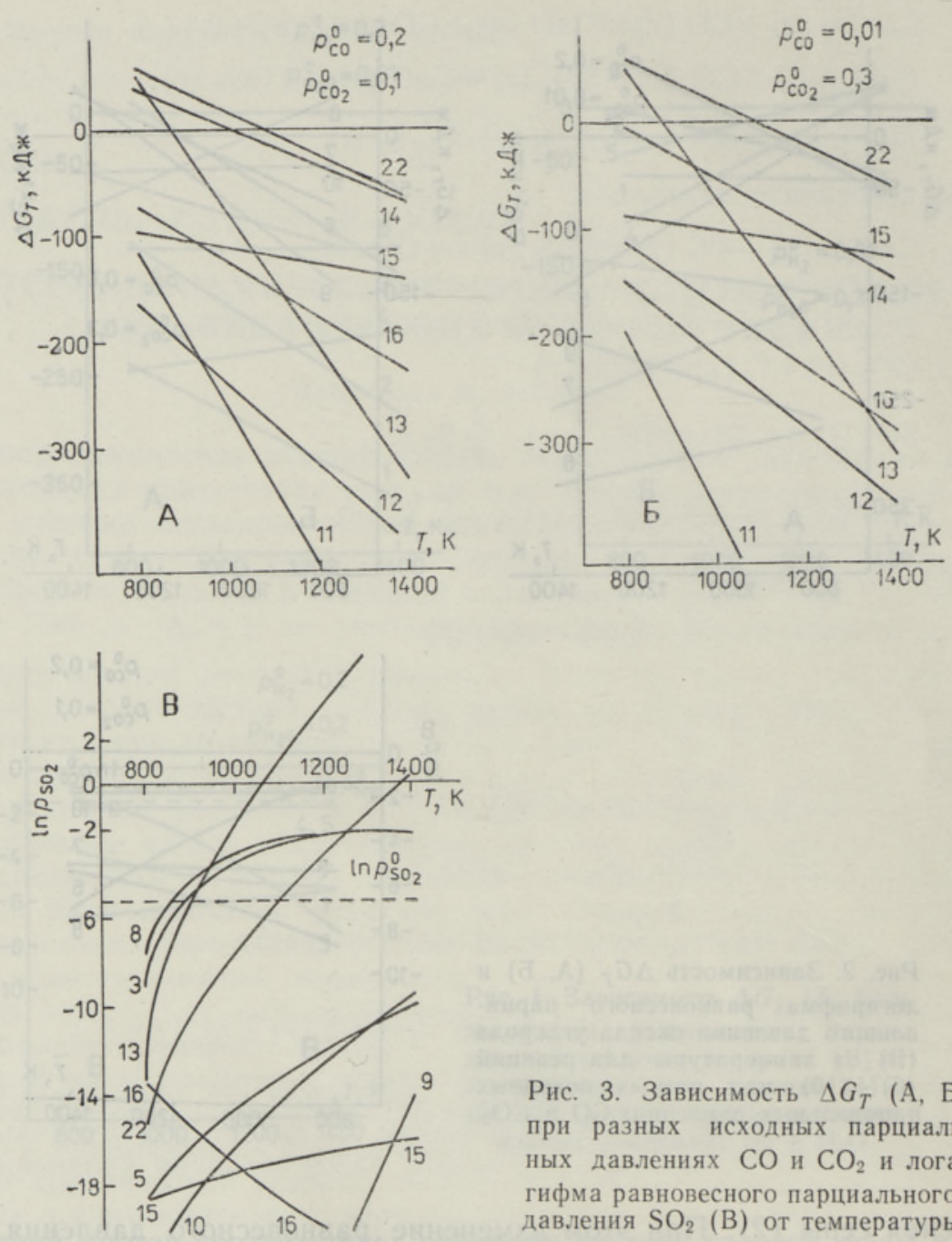

Рис. 3. Зависимость $\Delta G_{T}(\mathrm{~A}$, Б) при разных исходных парциальных давлениях $\mathrm{CO}$ и $\mathrm{CO}_{2}$ и логагифма равновесного парциального давления $\mathrm{SO}_{2}$ (B) от температуры.

низкие температуры способствуют протеканию реакции с образованием диоксида углерода, более высокие - оксида углерода. Вероятность реакции (11) с образованием оксида углерода возрастает также при уменьшении в реакционной смеси его парциального давления, тем самым повышается вероятность реакции (14), которая становится при $p_{\mathrm{Co}}^{0}=0,2$ возможной выше $1050 \mathrm{~K}$. При этом выделяется дополнительное количество оксида углерода, способ́твующего образованию сульфида кальция. Выше $900 \mathrm{~K}$ возможно протекание реакции восстановления сульфата кальция до его оксида (13), характеризующейся сравнительно высокой. температурной зависимостью $\Delta G_{\tau}$. Реакция взаимодействия сульфата кальция с сульфидом (22) становится возможной выше $1080 \mathrm{~K}$, но ее протекание при осуществлении процесса восстановления маловероятно из-за наличия термодинамически более выгодных конкурирующих реакций.

На рис. 3, В показаны температурные зависимости логарифмов равновесных давлений диоксида серы. Қак видно, наибольшие равновесные давления диоксида серы соответствуют реакциям (8), (3) и (13). При этом в наибольшей степени на выделение серы в газовую фазу в виде ее диоксида влияет реакция восстановления сульфата кальция углеродом (13), характеризующаяся наибольшей температурной зависимостью парциального равновесного давления диоксида серы. Все отмеченные реакции 

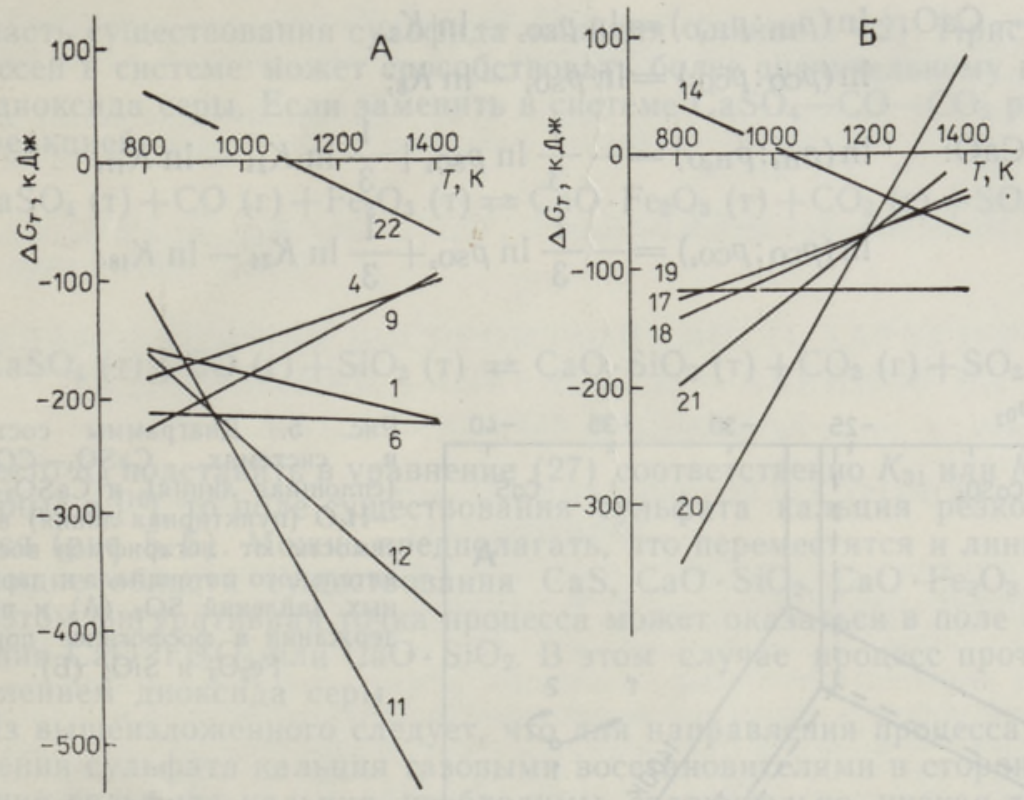

Puc. 4. Зависимость $\Delta G_{T}$ от температуры для реакций получения CaS (А) и окисления (Б).

возможны уже при сравнительно низких температурах - начиная с 850 - 950 K. Обратная реакция окисления сульфида кальция диоксидом углерода (9) в исследованных условиях является термодинамически невыгодной.

Итак, сильнейшим восстановителем сульфата кальция до сульфида по реакциям (11) и (12) в исследованных условиях является углерод (см. рис. $4, A)$. Величины $\Delta G_{\text {т }}$ для реакций (1) и (6) близкие. Поскольку восстановитсльные потенциалы $p_{\mathrm{CO}}^{0}: p_{\mathrm{CO}_{2}}^{0}$ и $p_{\mathrm{H}_{2}}^{0}: p_{\mathrm{H}_{2} \mathrm{O}}^{0}$ в реальных условиях в значительной мере колеблются, то трудно предположить, которая из этих реакций является превалирующей.

Изменение $\Delta G_{T}$ реакций окисления в изученной системе показано на рис. 4 , Б. До $1200 \mathrm{~K}$ термодинамически наиболее вероятным является окисление сульфида кальция по (20), выше этой температуры превалирует образование оксида кальция и диоксида серы (21). Однако в присутствии углерода, начиная с $1120 \mathrm{~K}$, предпочтительным становится окисление последнего по реакции (19). Выше $1200 \mathrm{~K}$ более вероятным в сравнении с окислением сульфида кальция является окисление газовых восстановителей. Таким образом, при проведении процесса в температурном интервале 1120 - $1200 \mathrm{~K}$ целесообразно введение в систему твердого восстановителя.

Для наглядности построены диаграммы состояния в системах $\mathrm{CaSO}_{4}$ $-\mathrm{H}_{2}-\mathrm{H}_{2} \mathrm{O}$ и $\mathrm{CaSO}_{4}-\mathrm{CO}-\mathrm{CO}_{2}$ по методике, предложсниой в [ ${ }^{8}$ ]. Для составления систем независимых уравнений выбраны лля первой системы реакции $(1),(3),(21)$ и $(17)$, для второй $-(6),(8),(21)$ и (18). Сопоставлением констант равновесия получены следующие уравнения прямых, разделяющих области существования соответствующих твердых компонентов реакций:

$\mathrm{CaSO}_{4}-\mathrm{CAS}: \ln \left(p_{\mathrm{H}_{2}}: p_{\mathrm{H}_{2} \mathrm{O}}\right)=-\frac{1}{4} \ln K_{1}$,

$$
\ln \left(p_{\mathrm{CO}}: p_{\mathrm{CO}_{2}}\right)=-\frac{1}{4} \ln K_{6}
$$




$$
\begin{aligned}
& \mathrm{CaSO}_{4}-\mathrm{CaO}: \ln \left(p_{\mathrm{H}_{2}}: p_{\mathrm{H}_{2} \mathrm{O}}\right)=\ln p_{\mathrm{SO}_{2}}-\ln K_{3} \text {, } \\
& \ln \left(p_{\mathrm{CO}}: p_{\mathrm{CO}_{2}}\right)=\ln p_{\mathrm{SO}_{2}}-\ln K_{8} \text {; } \\
& \ln \left(p_{\mathrm{CO}}: p_{\mathrm{CO}_{2}}\right)=-\frac{1}{3} \ln p_{\mathrm{SO}_{2}}+\frac{1}{3} \ln K_{21}-\ln K_{18}
\end{aligned}
$$

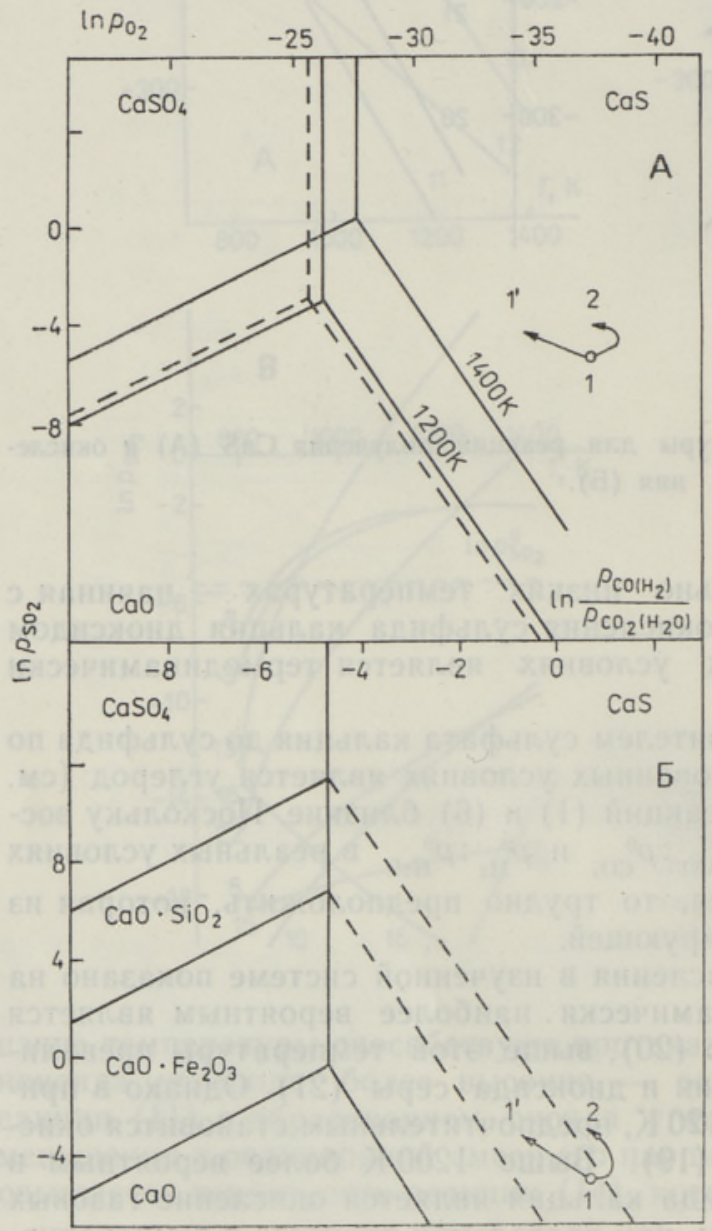

Рис. 5. Диаграммы состояния в системах $\mathrm{CaSO}_{4}-\mathrm{CO}-\mathrm{CO}_{2}$ (сплошная линия) и $\mathrm{CaSO}_{4}-\mathrm{H}_{2}-$ $-\mathrm{H}_{2} \mathrm{O}$ (пунктирная линия) в завигимости от логарифмов восстановительного потенщиала и парциальных давлений $\mathrm{SO}_{2}$ (А) и при содержании в фосфогипсе примесей $\mathrm{Fe}_{2} \mathrm{O}_{3}$ и $\mathrm{SiO}_{2}$ (Б).

На рис. 5, А представлены области существования $\mathrm{CaSO}_{4}, \mathrm{CaO}$ и $\mathrm{CaS}$ в зависимости от логарифма восстановительного потенциала газовой смеси и логарифма парциального давления диоксида серы при $1200 \mathrm{~K}$. Сплошные линии соответствуют восстановлению оксидом углерода, пунктирные - водородом. Видно, что с повышением температуры уменьшается область существования сульфида кальция и повышается вероятность выделения диоксида серы. При этом для водорода область существования сульфида кальция немного больше, чем для оксида углерода.

Поскольку в изучаемом нами реальном процессе возможно образование и диоксида серы, направление процесса описывается линией $1-1$ '. Фигуративная точка процесса при температуре $1200 \mathrm{~K}$ попадает в область существования оксида кальция при соотношении $p_{\mathrm{CO}}^{0}: p_{\mathrm{CO}_{2}}^{0}<0,02$. Соотношение $p_{\mathrm{Co}}: p_{\mathrm{CO}_{2}}$ определяет парциальное давление кислорода в системе по уравнению

$$
\ln p_{\mathrm{O}_{2}}=-2 \ln \left(p_{\mathrm{CO}}: p_{\mathrm{CO}_{2}}\right)-2 \ln K_{18}
$$

Введение в систему углерода уменьшает парциальное давление кислорода соответственно реакциям (14) и (19), поскольку в этом случае наблюдается наименьшее равновесное давление кислорода в системе. В результате этого выделяется дополнительное количество восстановителя - оксида углерода - и фнгуративная точка процесса глубже сдвигается 
в об̆ласть существования сульфида кальция (линия $1-2$ ). Присутствиё примесей в системе может способствовать более значительному выделению диоксида серы. Если заменить в системе $\mathrm{CaSO}_{4}-\mathrm{CO}-\mathrm{CO}_{2}$ реакцию (8) реакцией

$$
\mathrm{CaSO}_{4}(\mathrm{~T})+\mathrm{CO}(\mathrm{r})+\mathrm{Fe}_{2} \mathrm{O}_{3}(\mathrm{~T}) \rightleftarrows \mathrm{CaO} \cdot \mathrm{Fe}_{2} \mathrm{O}_{3}(\mathrm{~T})+\mathrm{CO}_{2}(\mathrm{r})+\mathrm{SO}_{2}(\mathrm{r})
$$

или

$$
\mathrm{CaSO}_{4}(\mathrm{~T})+\mathrm{CO}(г)+\mathrm{SiO}_{2}(\mathrm{~T}) \rightleftarrows \mathrm{CaO} \cdot \mathrm{SiO}_{2}(\mathrm{~T})+\mathrm{CO}_{2}(\mathrm{r})+\mathrm{SO}_{2}(\mathrm{r})
$$

и вместо $K_{8}$ подставить в уравнение $(27)$ соответственно $K_{31}$ или $K_{32}$, приведенные в [ $\left.{ }^{10}\right]$, то поле существования сульфата кальция резко уменьшается (рис. 5, Б). Можно предполагать, что переместятся и линии, разделяющие области существования $\mathrm{CaS}, \mathrm{CaO} \cdot \mathrm{SiO}_{2}, \mathrm{CaO} \cdot \mathrm{Fe}_{2} \mathrm{O}_{3}$ и $\mathrm{CaO}$. При этом фигуративная точка процесса может оказаться в поле существования $\mathrm{CaO} \cdot \mathrm{Fe}_{2} \mathrm{O}_{3}$ или $\mathrm{CaO} \cdot \mathrm{SiO}_{2}$. В этом случае процесс протекает с выделением диоксида серы.

Из вышеизложенного следует, что для направления процесса восстановления сульфата кальция газовыми восстановителями в сторону образования сульфида кальция необходимы сравнительно низкая температура (ниже $1200 \mathrm{~K}$ ) и высокий восстановительный потенциал реагентов. Более полному протеканию реакции содействует шихтовка к $\mathrm{CaSO}_{4}$ твердого восстановителя - углерода, повышающего восстановительный потенциал в результате связывания кислорода. В реальных системах возможно выделение диоксида серы при сравнительно низких температурах - выше 850 - 950 К. Поэтому в твердом продукте возможно незначительное содержание оксида кальция, а в газовую фазу выделяется немного диоксида серы, особенно в случае содержания в фосфогипсе примесей - полуторных оксидов. Для минимизации содержания оксида кальция в продукте необходимо применение более чистого фосфогипса, например, полученного при переработке кольского апатитового концентрата.

\section{ЛИТЕРАТУРА}

1. Владимиров П. С., Гаврилова Н. Ю. О термическом разложении сульфата кальция восстановительным газом, содержащим водород, окись углерода, водяной пар и двуокись углерода // Ж. прикл. хим., 1978, № 6, 1201-1205.

2. Влох В. М., Яворский В. Т., Мельник В. Ф. Термодинамические исследования процесса восстановления гипсов // Вопр. химии и хим. технол., 1975, вып. 37, $117-122$.

3. Волков А. Д. Термодинамическое исследование высокотемпературных процессов в системе $\mathrm{CaSO}_{4}+\mathrm{C} / /$ Тр. Ленингр. технол. ин-та, 1959, № 7, 150-153.

4. Смоленская Е. А., Кошкаров В. Я., Прохоров А. Г. Восстановление фосфогипса сернистым нефтяным коксом // Исследование окислительно-восстановительных процессов в оксидно-сульфидных и металлических системах. Свердловск, 1980, $108-115$.

5. Ченцов В. Н., Олейникова T. В., Епифанов В. С. Термодинамические основы конверсии фосфогипса в сернистый ангидрид и известь // НПО «Минудобрения» НИУИФ. М., 1983, 21 с. Деп. в ВИНИТИ № 19-хп-Д84.

6. Ченцсо В. Н., Олейникова Т. В., Епифанов В. С., Хрящев С. В. Термодинамическое исследование процесса восстановительного разложения сульфата кальция // Ж. прикл. хим., 1983, № 5, 983-986.

7. Грәнчаров Н., Кирилов П., Пеловски И., Домбалов И., Виденов Н. Термодинами. чен анализ на термохимичното разлагане на фосфогипса при различни парциални налягания на газовите компоненти // Химия и инд., 1983, № 1, 27-29.

8. Рогачев О. В., Костыльков Н. Г. Анализ разногласий термодинамических расчетов и практики восстановительного разложения ангидрита / ГИГХС. М., 1985. 28 с. Деп. в ВИНИТИ № 468-хп-Д85. 
9. Тิюрн Л. И., Тинт П. А., Кярблане Э. Х. Равновесие в системе сульфат кальция-мйнеральные примеси-восстановители // Тр. Таллин. политехн. ин-та, 1983, вып. $542,75-83$.

10. Кярблане Э. Х., Кууск А. А.-М., Куусик $P$. О. Термодинамический анализ процесса высокотемпературной обработки фосфогипса // Тр. Таллин. политехн. ин-та, 1980, вып. $479,21-28$.

Таллиннский политехнический институт

Поступила в редакцию

19/XII 1988

\section{A. TRIKKEL, L. TURN, R. KUUSIK}

\section{FOSFOKIPSIST KALTSIUMSULFIIDI SAAMISE TERMODUNAAMILISEST TÕENÄOSUSEST}

Fosfokipsist kaltsiumsulfiidi saamise võimaluste hindamiseks uuriti 22 reaktsiooni kulgemise termodünaamilist tõenäosust temperatuurivahemikus $800-1400 \mathrm{~K}$ järgmistes süsteemides: $\mathrm{CaSO}_{4}-\mathrm{H}_{2}-\mathrm{H}_{2} \mathrm{O}, \mathrm{CaSO}_{4}-\mathrm{CO}-\mathrm{CO}_{2}$ ja $\mathrm{CaSO}_{4}-\mathrm{C}-\mathrm{O}_{2}$. Reaktsioonide võimalikkust analüüsiti Gibbsi energia muutumise pôhjal, arvestades gaasiliste reagentide osarōhkusid süsteemis, samuti reaktsiooniproduktide tasakaalurõhkude ning tahkete komponentide olekudiagrammide järgi. Leiti, et protsessi mõjutamiseks CaS tekke suunas on vajalik suhteliselt madal temperatuur - alla $1200 \mathrm{~K}$, kōrge taandajate kontsentratsioon gaasifaasis, tahke taandaja lisamine kaltsiumsulfaadile ning madala lisandite sisaldusega fosfokipsi kasutamine.

\section{A. TRIKKEL, L. TURN, R. KUUSIK}

\section{THERMODYNAMICAL PROBABILITY OF GETTING CALCIUM SULPHIDE FROM PHOSPHOGYPSUM}

The probability of getting $\mathrm{CaS}$ from phosphogypsum in the temperature range of $800-1400 \mathrm{~K}$ on the basis of 22 reactions in the systems $\mathrm{CaSO}_{4}-\mathrm{H}_{2}-\mathrm{H}_{2} \mathrm{O}, \mathrm{CaSO}_{4}-$ $\mathrm{CO}-\mathrm{CO}_{2}$ and $\mathrm{CaSO}_{4}-\mathrm{C}-\mathrm{O}_{2}$ has been determined by means of a thermodynamic analysis. The probability of the reactions has been estimated by the equilibrium partial pressures of the gaseous products, by the state diagrams of the solid phase and by the change of the Gibbs energy, taking into consideration the real partial pressures of the. gaseous reagents. Lowering the temperature below $1200 \mathrm{~K}$ and increasing the reduction potential of the gaseous reductants leads to an increase in the yield of $\mathrm{CaS}$. The addition of solid reductant and the use of pure phosphogypsum with minimum content of impurities is recommendable. 